\title{
Internacionalización de empresas latinas: evolución y tendencias
}

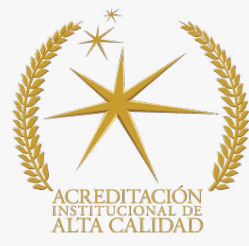

\section{Latin companies' internationalization: evolution and trends}

Artículo de revisión.

Fecha de recepción: 03/06/2020

Fecha de devolución: 24/07/2020

Fecha de aceptación: 31/07/2020

Fecha de publicación: 25/09/2020

\section{Pedro Luis Duque Hurtado \\ Universidad Católica Luis Amigó. \\ Manizales, Caldas (Colombia) \\ pedro.duquehu@amigo.edu.co}

\section{Oscar Eduardo Meza Aguirre}

Universidad Nacional de Colombia /

Universidad Católica Luis Amigó.

Manizales, Caldas (Colombia)

oemezaa@unal.edu.co

oscar.mezaag@amigo.edu.co

\section{Gastón Adolfo Zapata Lesmes \\ Universidad Católica Luis Amigó. \\ Manizales, Caldas (Colombia) \\ gaston.zapatale@amigo.edu.co}

\section{José David Giraldo Castellanos \\ Universidad Católica Luis Amigó. \\ Manizales, Caldas (Colombia) \\ Jose.giraldoas@amigo.edu.co}

Para citar este artículo:

Duque, P., Meza, O., Zapata, G. \& Giraldo, J. (2021)

Internacionalización de empresas latinas: evolución y tendencias. Económicas CUC, 42(1), 122-152. DOI: https://doi.org/10.17981/econcuc.42.1.2021.Org.1

\section{Resumen}

El propósito de este estudio es realizar un análisis sistémico de las investigaciones sobre internacionalización de empresas latinas. Para ello, se efectúa un análisis de red complementado con herramientas bibliométricas, logrando así, la identificación de las perspectivas del tema. Inicialmente, se efectuó la búsqueda temática en las bases de datos WoS y Scopus; las referencias obtenidas fueron procesadas mediante bibliometrix y Gephi, facilitando la clasificación de los documentos según su relevancia en tres categorías, clásicos, estructurales y recientes, finalmente se realiza un análisis de cocitaciones. Este último procedimiento permitió identificar cuatro perspectivas principales en el área: diversificación y desempeño, emprendimiento, exportaciones y mercado, estrategias y competencia.

Palabras clave: Internacionalización; empresas latinas; competitividad; diversificación; economía emergente; mapeo científico

\section{Abstract}

The purpose of this study is to conduct a systemic analysis of research on the internationalization of Latin American companies. For this purpose, network analysis is carried out, complemented with bibliometric tools, thus achieving the identification of the perspectives on the subject. Initially, a thematic search was done in the WoS and Scopus databases; the references obtained were processed through bibliometrix and Gephi, facilitating the classification of the documents according to their relevance in three categories, classical, structural and recent, and finally, a quotation analysis is made. This last procedure allowed the identification of four main perspectives in the area: diversification and performance, entrepreneurship, exports and market, strategies, and competition.

Keywords: Internationalization; Latin American firms; competitiveness; diversification; emerging economy; scientific mapping

JEL: F23. 


\section{INTRODUCCIÓN}

La internacionalización de las empresas es un proceso mediante el cual se desarrollan actividades de compra-venta en el exterior (Aguilera, Ciravegna, CuervoCazurra \& Gonzalez-Pérez, 2017), de tal manera que se convierte en un aspecto esencial para su crecimiento y sus perspectivas futuras de supervivencia (Johanson \& Vahlne, 1990). En conjunto con las dinámicas de expansión y diversificación de riesgos comerciales, de mercado y de aprovisionamiento, determinados por la toma de decisiones corporativas orientadas a la adquisición y ubicación de activos estratégicos adoptados por multinacionales de países desarrollados, a modelos gradualistas utilizados por empresas de menor envergadura y pymes de países emergentes (Johanson \& Vahlne, 1977). Las empresas de estos mercados deben sortear mayores dificultades en el proceso de internacionalización, por lo cual, inician con exportaciones ocasionales en emprendimientos fortuitos y pasan a exportaciones regulares en emprendimientos planificados.

El entorno empresarial de la actualidad exige a las firmas ir más allá del uso de enfoques clásicos unidimensionales de proveeduría-procesos productivos y entrega a clientes, en donde la organización actúa sola, y no dentro de las disrupciones estratégicas en el imperativo de generación de redes de relaciones (Johanson \& Vahlne, 2015), favoreciendo la innovación en estrategias de ingresos, de portafolio y en las habilidades gerenciales propias del proceso de expansión internacional.

La internacionalización de las empresas latinas ha sido un tema poco explorado, y en comparación con el conocimiento que se cuenta de otras regiones, este resulta aún limitado (Aguilera et al., 2017). A pesar de la relevancia del tema, a la fecha, no se identifico revisión que muestre su desarrollo y que plantee sus perspectivas o subáreas. A partir de la búsqueda en las bases de datos, se identificaron algunas aproximaciones, por ejemplo, Yaprak \& Karademir (2010) realizan una revisión crítica de la literatura sobre la internacionalización de los grupos empresariales en los mercados emergentes. Aguilera et al. (2017) analizan la naturaleza y el comportamiento de las empresas latinas que se han posicionado en mercados internacionales. En consecuencia, el presente artículo pretende realizar un análisis sistémico de las investigaciones sobre internacionalización de empresas latinas, a través de un análisis de red, apoyado en herramientas bibliométricas que permiten la identificación de las perspectivas del tema.

Para cumplir con este objetivo, primero se efectuó la consulta del tema en las bases de datos Scopus y Web of Science (WoS), teniendo en cuenta el periodo de tiempo comprendido entre los años 2000 y 2019. Los resultados fueron exportados y procesados mediante herramientas como bibliometrix, $\mathrm{R}$ y Gephi. Posteriormente, se realiza un estudio bibliométrico, se clasifican y analizan los documentos más relevantes bajo la analogía del árbol (raíces, tronco y hojas). Finalmente, se determinaron a través de un análisis de cocitaciones las perspectivas o subáreas en las que se desarrolla la investigación de internacionalización de empresas latinas. 
El documento se compone de tres apartados. El primero detalla la metodología empleada para la búsqueda, elección y procesamiento de los artículos objeto de estudio. En la segunda, se plantea el desarrollo de la investigación, análisis y hallazgos. Finalmente, en la tercera se exponen las conclusiones, limitaciones y recomendaciones para posteriores investigaciones.

\section{Metodología}

La metodología de la presente investigación se desarrolló en tres etapas. Primero, se analizó la importancia del tema, para lo cual se tienen en cuenta indicadores bibliométricos y se emplea la herramienta bibliometrix que facilita el mapeo científico (Aria \& Cuccurullo, 2017). Segundo, se construye la red social de los documentos más relevantes en el área, y utilizando la analogía del árbol se clasifican y examinan. Finalmente, en la tercera etapa utilizando el análisis de co-citaciones se identifican subáreas de trabajo.

\section{Paso 1: Importancia del Tema}

En esta etapa se emplean cuatro indicadores bibliométricos como lo sugieren Zupic y Čater (2015) para analizar la producción científica en el área. El primer análisis, compara la producción anual registrada en las bases de datos WoS y Scopus, el segundo, presenta el top 10 de los países con mayor número de publicaciones en el campo, el tercero, muestra los autores más relevantes, y el cuarto, relaciona las revistas con mayor número de publicaciones del tema.

Los parámetros de la búsqueda se relacionan en la Tabla 1 (La fecha de la consulta fue el 4 de enero de 2020).

TABLA 1.

Criterios y resultados de búsqueda.

\begin{tabular}{llll}
\hline \multicolumn{1}{c}{ Base de datos } & \multicolumn{1}{c}{ Ecuación de búsqueda } & Resultados & Artículos totales \\
\hline Scopus & $\begin{array}{l}\text { TEMA: } \\
\text { "internationalization*” AND "latin*” AND }\end{array}$ & 131 & 225 \\
Web of Science & $\begin{array}{l}\text { ("enterprise*" OR "business*) } \\
\text { Periodo de tiempo: 2000-2020 }\end{array}$ & 94 \\
\hline
\end{tabular}

Fuente: Elaboración propia.

Para el análisis bibliométrico se utiliza Bibliometrix, el cual es código desarrollado por Aria y Cuccurullo (2017) y explicada en el documento "bibliometrix: An R-tool for comprehensive science mapping analysis" de su autoría, esta herramienta ha sido empleada en múltiples investigaciones de diversas disciplinas que utilizan cartografía científica (Taebi Javid, Nazari \& Ghaeli, 2019; Pourkhani, Abdipour, Baher \& Moslehpour, 2019; Puck \& Filatotchev, 2018; Tani, Papaluca \& Sasso, 2018). 


\section{Paso 2: Red y Árbol}

Para la generación de la red se empleó la teoría de grafos, la cual muestra los documentos y la manera en que se relacionan. Este método ha sido desarrollado y aplicado en investigaciones en las ciencias sociales (Wallis, 2007; Wasserman \& Faust, 2012; Yang, Keller \& Zheng, 2016). Esta técnica permite generar información asociada a la tipología y características de la red y de cada documento que la integra.

El primer paso de este procedimiento es obtener los registros de las bases de datos WoS y Scopus, luego se combinan y se extrae las referencias (bibliografía) a cada uno de los documentos, posteriormente, se eliminan los datos duplicados y se realiza la transformación de estos datos en una red, para esto se emplea la herramienta Sci2 (Indiana University and SciTech Strategies, 2009).

El segundo paso, consiste en la visualización y análisis de la red, para ello se utiliza el aplicativo Gephi (Mathieu, Sebastien \& Mathieu, 2009), esta herramienta ha sido validada en otras investigaciones (Duque \& Cervantes-Cervantes, 2019; Ferguson, 2012; Jacomy, Venturini, Heymann \& Bastian, 2014). Posteriormente, en esta misma herramienta se calculan los indicadores Indegree, Outdegree y betweenness de cada documento que hace parte de la red. El Indegree muestra el número de veces que un documento ha sido referenciado por otros; el Outdegree, indica el número de veces que un nodo en particular cita a otros, también se puede considerar como el número de conexiones que se desprenden de cada documento (Wallis, 2007); y el Betweenness, representa el grado de intermediación y centralidad de cada elemento al interior de la red (Freeman, 1977), básicamente este indicador muestra que el documento cita y ha sido citado por los demás (Zhang \& Luo, 2017), para evidenciar su relevancia y aceptación en la temática de estudio.

La red resultante de los procesos mencionados anteriormente está compuesta por 744 documentos, y a cada documento se le generan los 3 indicadores mencionados, clasificándolos a través del esquema propuesto por Robledo, Osorio y López (2014), en el cual mediante la analogía de un árbol se organizan los documentos en 3 categorías: La primera denominada raíz (alto indegree), allí se localizan los documentos hegemónicos o también llamados clásicos, estos son citados pero no citan a otros documentos de la red; la segunda, llamada tronco (alto betweenness), involucra a los documentos conocidos como estructurales, estos citan y a la vez son citados por otros; y la tercera, conocida como hojas (alto outdegree) asocia a los documentos más recientes, su principal característica es que citan a los demás (tronco y raíces) pero no son referenciados. Esta metodología es sustentada en diversos trabajos (Buitrago, Duque \& Robledo, 2020; Duque \& Cervantes-Cervantes, 2019; Landínez, Robledo \& Montoya, 2019; Marín, Robledo \& Duque-Méndez, 2017; Camargo, Cardona \& Mira, 2017; Zuluaga et al., 2016).

Para realizar la revisión bibliográfica se eligieron los documentos más relevantes (los de más altos indicadores) de cada categoría (Tabla 4). 


\section{Paso 3: Perspectivas}

Para identificar las perspectivas o subáreas del tema en la red generada en la etapa anterior, se aplicó el algoritmo de clusterización propuesto por Blondel, Guillaume, Lambiotte y Lefebvre (2008). Esta técnica permite a través de un análisis de co-citaciones categorizar los documentos. Finalmente, mediante minería de texto se identifican los temas que componen las perspectivas, para ello se emplea el aplicativo R, específicamente el paquete WordCloud (Ohri, 2012). Ver tabla 5.

\section{Resultados y Discusión}

\section{Producción Mundial}

En la Figura 1 se relaciona la producción mundial de documentos científicos en las bases de datos WoS y Scopus asociados al tema, publicados entre los años 2000 y 2019. Hasta el año 2014 se habían publicado el 39\% del total de investigaciones en el área, sin embargo, la mayor producción se logra a partir del año 2015, en los últimos 5 años se publicaron más de 120 artículos, lo que representa cerca de $60 \%$. Como lo muestra la línea de tendencia, el interés de la comunidad científica en el tema ha aumentado a través del paso del tiempo, lo que se refleja en una tasa de crecimiento anual del 18\%. A pesar que el número de publicaciones en Scopus es mayor que en WoS, el comportamiento y tendencia en las dos es similar. (Figura 1 y Figura 2).

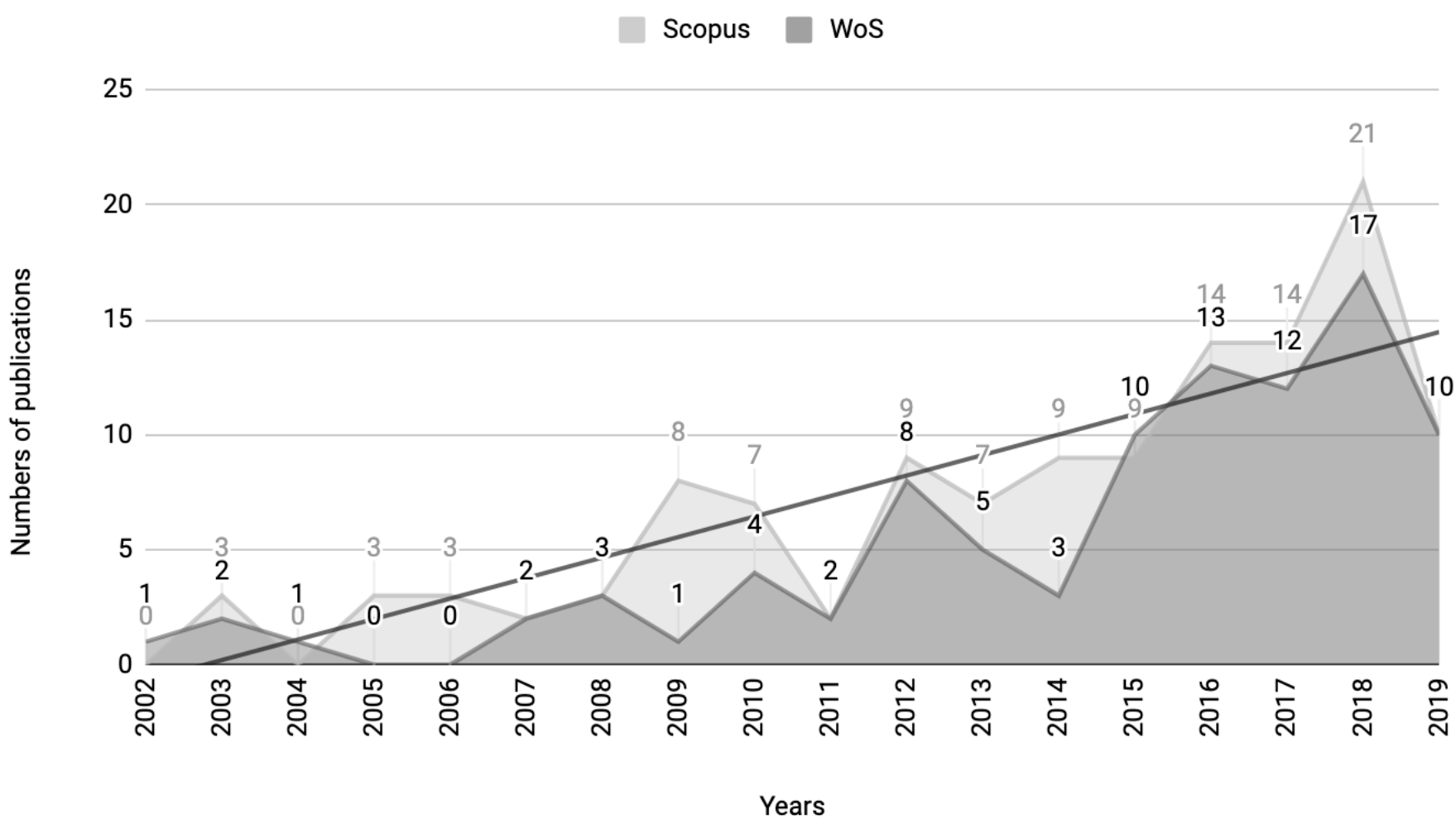

Figura 1. Producción científica anual.

Fuente: Elaboración propia. 
Al efectuar el análisis de la producción académica del tema por países y regiones, se identifica que Estados Unidos lidera el listado con 59 publicaciones, seguido por España con 45, y en tercer lugar se encuentra Colombia con 32. A pesar de que la relevancia del tema para la Latinoamérica, tan solo el $47 \%$ de las publicaciones se vinculan a países de la región (Colombia, Brasil, Chile, Costa Rica, México, Argentina).

40

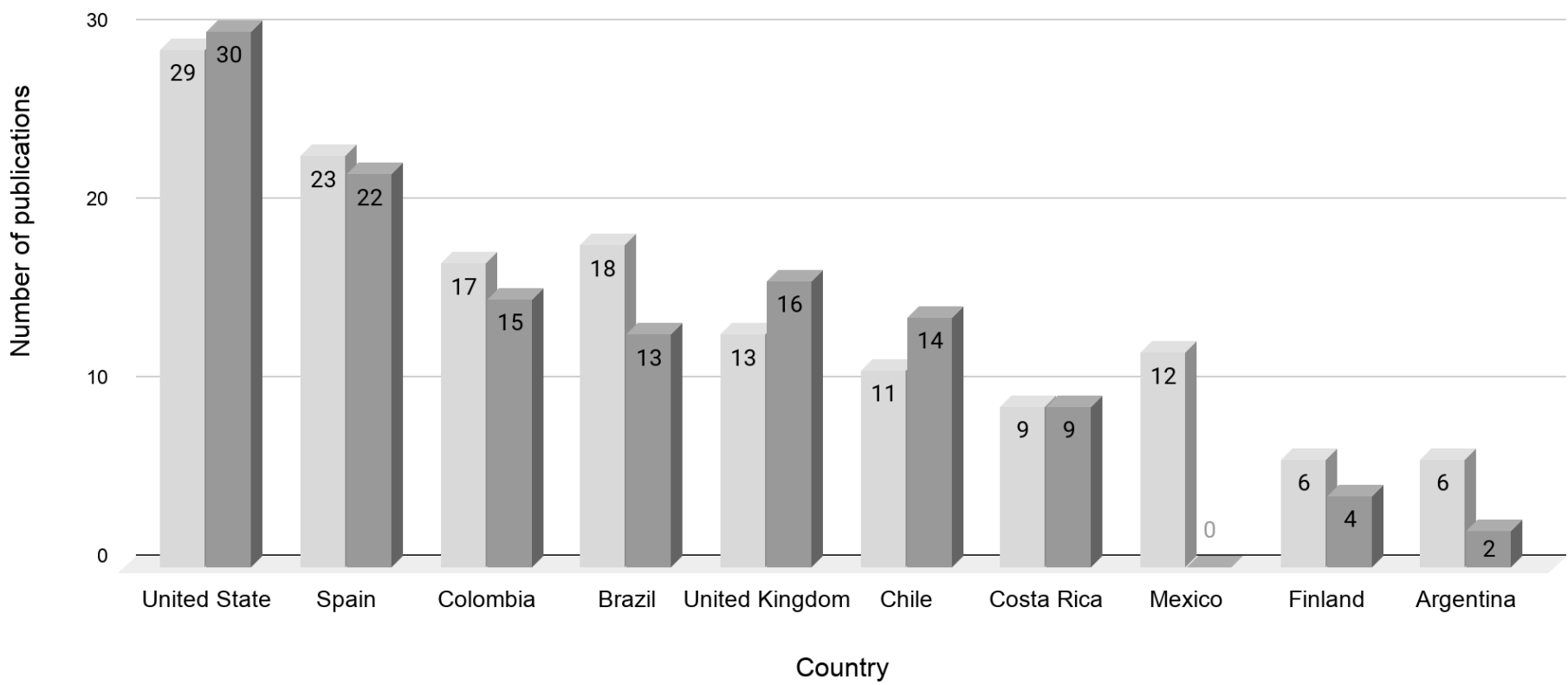

Figura 2. Top 10 países.

Fuente: Elaboración propia.

\section{Principales Autores y Revistas}

La Tabla 2 presenta la relación de los 10 autores más relevantes del área, considerando el número de publicaciones vinculadas al tema como el factor clasificatorio, y vinculado el indicador de caracterización de nivel de producción propuesto por Hirsch (2005), conocido como h-index. Luciano Ciravegna vinculado al King's College London es el autor con mayor número de publicaciones tanto en WoS como en Scopus, en segunda posición en la base de datos Scopus se encuentra María Alejandra Gonzales Pérez de la Universidad EAFIT en Colombia, mientras que esta posición en WoS la ocupa Alvaro Cuervo Cazurra de Northeastern University en Estados Unidos. 
TABLA 2.

Autores más relevantes.

\begin{tabular}{llllll}
\hline \multicolumn{1}{c}{ Scopus } & & & \multicolumn{2}{l}{ WoS } \\
\hline \multicolumn{1}{c}{ Autor } & $\begin{array}{c}\text { Número de } \\
\text { publicaciones }\end{array}$ & H-Index & \multicolumn{1}{c}{ Autor } & $\begin{array}{c}\text { Número de } \\
\text { publicaciones }\end{array}$ & H-Index \\
\hline Ciravegna L. & 7 & 20 & Ciravegna L. & 7 & 20 \\
Gonzales-Perez MA. & 6 & 13 & Cuervo-Cazurra A. & 4 & 36 \\
Brenes ER. & 4 & NR & Newburry W. & 4 & 26 \\
Borda A. & 3 & NR & Bianchi C. & 3 & 28 \\
Carneiro J. & 3 & 17 & Borda A. & 3 & NR \\
Cuervo-Cazurra A. & 3 & 36 & Brenes ER. & 3 & NR \\
Larios Hernández GJ. & 3 & 3 & Carneiro J. & 3 & 17 \\
Newburry W. & 3 & 26 & Felzensztein C. & 3 & 23 \\
Alzaraz J. & 2 & NR & Gonzales-Perez MA. & 3 & 13 \\
Bianchi C. & 2 & 28 & Kundu SK. & 3 & 28 \\
\hline
\end{tabular}

Fuente: Elaboración propia.

En la Figura 3 se identifican tres clusteres o grupos de cocitaciones, el primero y más grande está integrado por Cuervo Cazurra, Khanna T., Luo YD., Dunning JH. entre otros, siendo el primero de la lista el autor con mayor número de citaciones en la red, esto se ve reflejado en su h-index (37 en google citations), el cual es el más alto de los autores relevantes en el campo. El segundo, compuesto por Johanson J., Eisenhardt KM. y Ciravegna L. este último el investigador con mayor número de publicaciones relacionadas al tema. Finalmente, el tercer y más pequeño de los clusteres está representado por Perezbatres LA., Brenes EA., entre otros.

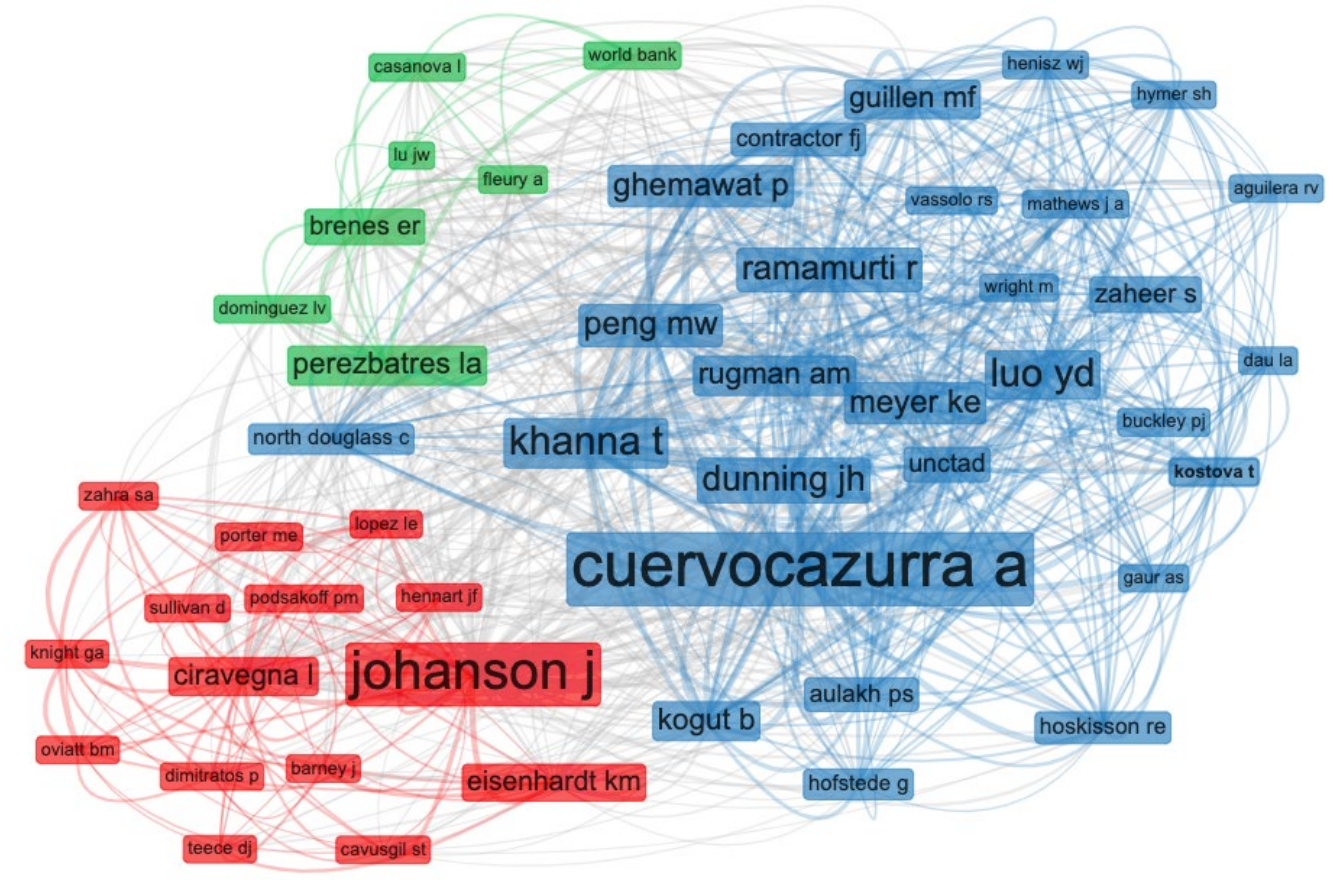

Figura 3. Red de co-citaciones.

Fuente: Elaboración propia. 
La revista con el mayor número de publicaciones asociados al tema es Journal of Business Research, ésta se encuentra indexada en WoS y Scopus, en ambas bases de datos hace parte del cuartil 1. La única revista latinoamericana con presencia en este Top 10 es la colombiana Innovar, la cual se encuentra en cuartil 3 en Scopus, sin embargo, no tiene visibilidad en WoS.

TABLA 3.

Revistas más relevantes.

\begin{tabular}{llll}
\hline \multicolumn{1}{c}{ Revista } & $\begin{array}{c}\text { Número de } \\
\text { publicaciones }\end{array}$ & $\begin{array}{c}\text { Cuartil } \\
\text { Journal of Business Research }\end{array}$ & $\begin{array}{c}\text { Base de } \\
\text { datos }\end{array}$ \\
\hline Academia Revista Latinoamericana de Administración & 9 & Q1 & Scopus \\
& 14 & Wos & Scopus \\
Journal of World Business & 7 & Q4 & Wos \\
Journal of International Management & 4 & Q1 & Scopus \\
Review of International Business and Strategy & 7 & Q1 & Scopus \\
European Business Review & 3 & Q2 & Wos \\
Journal of International Business Studies & 4 & Q2 & Scopus \\
Journal of Small Business And Entreprise Development & 3 & NC & Wos \\
International Marketing Review & 3 & Q2 & Scopus \\
\hline & 3 & NC & Wos \\
& 2 & & Scopus \\
& 2 & Q1 & Wos \\
\hline & 2 & Q2 & Scopus \\
& 3 & NC & WoS \\
& 2 & Q1 & Scopus \\
& 32 & WoS \\
& 2 & Q3 & Scopus \\
\hline
\end{tabular}

Fuente: Elaboración propia.

\section{Análisis de la Red}

Los resultados generados por la búsqueda en las bases de datos permitieron obtener una red compuesta por 744 nodos (documentos) y 2133 aristas (enlaces o referencias entre los documentos). Los cuatro grupos principales (en adelante perspectivas) reúnen el $64 \%$ de los documentos. El tamaño de los nodos indica el número de citaciones recibidas (Figura 4). 


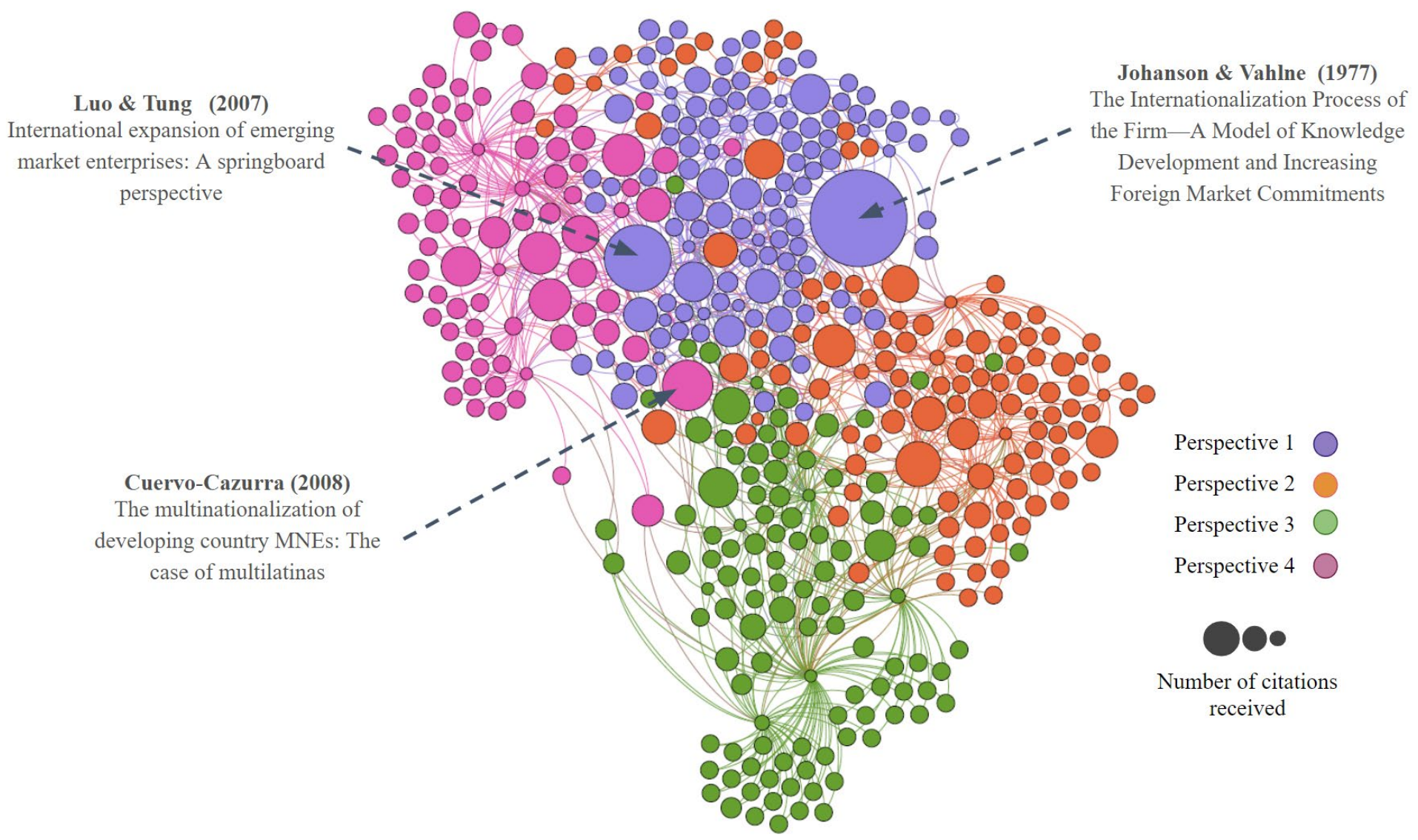

Figura 4. Grafo internacionalización empresas latinas.

Fuente: Elaboración propia.

Los tres documentos más relevantes area, y considerados como hegemónicos de la red se describen a continuación:

- "The Internationalization Process of the Firm-A Model of Knowledge Development and Increasing Foreign Market Commitments" de Johanson y Vahlne (1977), en este artículo los autores proponen un modelo de internacionalización para las empresas, centrado en la adquisición y uso gradual del conocimiento de los mercados extranjeros, este enfoque es conocido como el modelo Uppsala. Este documento registra más de 14.000 citaciones (google citations).

- "International expansion of emerging market enterprises: A springboard perspective" de Luo y Tung (2007) es el segundo documento más citado al interior de la red, en éste, los autores proponen un nuevo modelo de internacionalización de la empresa, el cual se sustenta en el conocimiento pleno del mercado, adquisición de activos estratégicos e integración con las industrias locales.

- Finalmente, el tercer documento es "Transforming disadvantages into advantages: developing-country MNEs in the least developed countries" de CuervoCazurra y Genc (2008). En este artículo se aborda el análisis comparativo de las ventajas y desventajas de empresas multinacionales de países en desarrollo en contraste con las de países desarrollados. A través de un estudio empírico exponen como las difíciles condiciones institucionales y gubernamentales en las que se encuentran los países en desarrollo, les permite convertir dicha desventaja en 
una ventaja en el comercio internacional y en su proceso de internacionalización, dado que ya tienen experiencia enfrentando un contexto más hostil para el modelo de negocio.

\section{Principales documentos (Árbol)}

Para ilustrar la analogía del árbol, descrita en el apartado metodológico se presenta la Figura 5, ésta representa el árbol del campo de estudio, ubicando los documentos dependiendo su categoría, raíces, tronco y hojas, estas últimas divididas en las cuatro perspectivas o sub áreas identificadas.

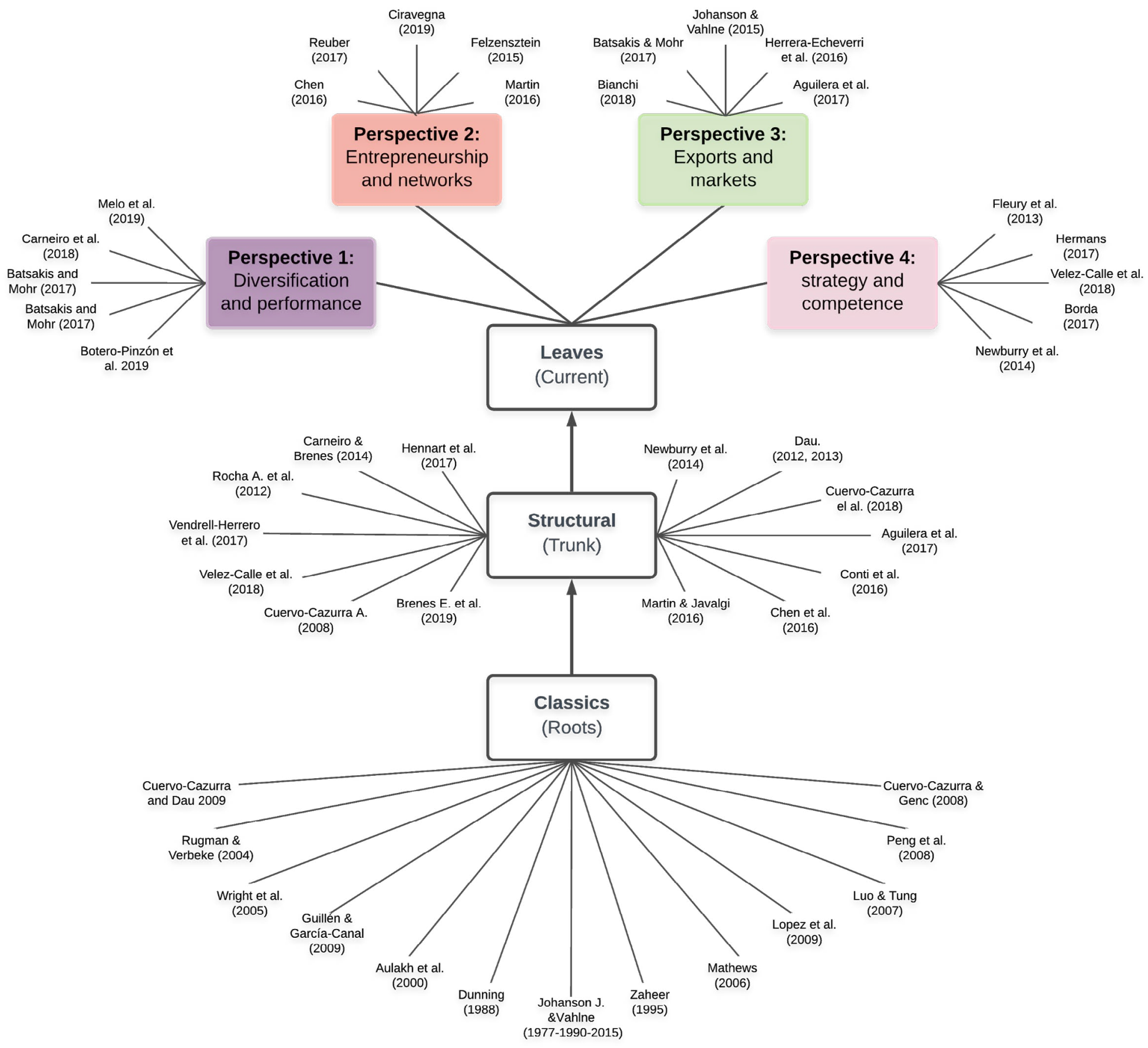

Figura 5. Árbol internacionalización de empresas latinas.

Fuente: Elaboración propia. 
TABLA 4.

Documentos principales

\begin{tabular}{ccc}
\hline $\begin{array}{c}\text { Hegemonic } \\
\text { (Root) }\end{array}$ & $\begin{array}{c}\text { Structural } \\
\text { (Trunk) }\end{array}$ & $\begin{array}{c}\text { Currents } \\
\text { (Leafs) }\end{array}$ \\
\hline
\end{tabular}

The Internationalization Process of the Firm-A Model of Knowledge Development and Increasing Foreign Market Commitments (Johanson \& Vahlne, 1977).

The Eclectic Paradigm of International Production: A Restatement and Some Possible Extensions (Dunning, 1988).

The Mechanism of Internationalisation (Johanson \& Vahlne, 1990).

Overcoming the Liability of Foreignness (Zaheer, 1995).

Export Strategies and Performance of Firms from Emerging Economies: Evidence from Brazil, Chile, and Mexico (Aulakh et al., 2000).

A perspective on regional and global strategies of multinational enterprises (Rugman \& Verbeke, 2004b).

Strategy Research in Emerging Economies: Challenging the Conventional Wisdom (Wright et al., 2005).

Dragon multinationals: New players in 21st century globalization (Mathews, 2006).
The multinationalization of developing country MNEs: The case of multilatinas (CuervoCazurra, 2008).

The international commitment of lateinternationalizing Brazilian entrepreneurial firms (Da Rocha et al., 2012).

Pro-market reforms and developing country multinational corporations (Dau, 2012).

Learning across geographic space: Pro-market reforms, multinationalization strategy, and profitability (Dau, 2013).

Employer Attractiveness in Latin America:

The Association

Among Foreignness, Internationalization and Talent Recruitment (Newburry et al., 2014).

Latin American firms competing in the global economy (Carneiro \& Brenes, 2014).

Entrepreneurial orientation, marketing capabilities and performance: The Moderating role of Competitive Intensity on Latin American International New Ventures (Martin \& Javalgi, 2016).

When distance does not matter: Implications for Latin American multinationals (Conti et al., 2016).
Motives as Locational Determinants: A Study of FDI between India and LAC (Varma et al., 2015).

Export behavior and board independence in Colombian family firms: The reverse causality relationship (Herrera-Echeverri et al., 2016).

Cross-national uncertainty and level of control in cross-border acquisitions: A comparison of Latin American and U.S. multinationals (Malhotra et al., 2016).

The impact of business group diversification on emerging market multinationals: Evidence from Latin America (Wu, Pangarkar \& Wu, 2016).

Revisiting the relationship between product diversification and internationalization process in the context of emerging market MNEs (Batsakis \& Mohr, 2017).

Looking for a service opening: Building reputation by leveraging international activities and host country context (Borda et al., 2017).

SME international performance in Latin America: The role of entrepreneurial and technological capabilities (Bianchi et al., 2017).

Formal and informal institutions and the expatriation assignment: The case of Japanese subsidiaries in Latin America (Moreira \& Ogasavara, 2018). 
International expansion of emerging market enterprises: A springboard perspective (Luo \& Tung, 2007).

Transforming disadvantages into advantages: developingcountry MNEs in the least developed countries (CuervoCazurra \& Genc, 2008).

An institution-based view of international business strategy: a focus on emerging economies (Peng et al., 2008).

Born global or born regional? Evidence from an exploratory study in the Costa Rican software industry (López et al., 2009).

The American Model of the Multinational Firm and the "New" Multinationals From Emerging Economies (Guillén \& García-Canal, 2009).

Promarket Reforms and Firm profitability in Developing Countries (Cuervo-Cazurra \& Dau, 2009).

The Uppsala internationalization process model revisited: From liability of foreignness to liability of outsidership (Johanson \& Vahlne, 2015).
Internationalization and value orientation of entrepreneurial ventures-a Latin American perspective (Chen et al., 2016).

Openness, international champions, and the internationalization of Multilatinas (Hennart et al., 2017).

Multilatinas and the internationalization of Latin American firms (Aguilera et al., 2017).

Building international business bridges in geographically isolated areas: The role of foreign market focus and outward looking competences in Latin American SMEs (Vendrell-Herrero et al., 2017).

Home country uncertainty and the internationalizationperformance relationship: Building an uncertainty management capability (Cuervo-Cazurra et al., 2018).

Internationalization and performance: the role of depth and breadth (VélezCalle et al., 2018).

Managing institutional voids: A configurational approach to understanding high performance antecedents (Brenes et al., 2019).
Internationalisation commitment of emerging market firms: A comparative study of Chile and Brazil (Bianchi et al., 2018).

Organizational slack as an enabler of internationalization: The case of large Brazilian firms (Carneiro et al., 2018).

Latin American franchise internationalization: The impact of institutional environment (De Resendre et al., 2019).

Aztec multilatinas: characteristics and strategies of Mexican multinationals (Huesca-Dorantes et al., 2018).

The internationalization of Nigerian firms: Motivations and location patterns (Omokaro-Romanus et al., 2019).

The timing of internationalization Drivers and outcomes (Ciravegna et al., 2019).

The international profile of the firm: Application of latent profile analysis to the case of Colombia (Botero-Pinzón et al., 2019).

Fuente: Elaboración propia.

\section{Documentos clásicos (raíz)}

Los documentos considerados como clásicos o hegemónicos son investigaciones que soportan la teoría sobre la internacionalización empresarial, concentran su orientación de estudio en el análisis de los diferentes modelos y factores involucrados en los procesos de apertura de mercados exteriores. Como el principal modelo teórico abordado entre las diferentes fuentes empleadas, se encuentra el modelo de Uppsala 
(Johanson \& Vahlne, 1990, 1977, 2015; López, Kundu, \& Ciravegna, 2009). De este modelo se presenta una caracterización de los elementos y etapas que comprende, para luego establecer un contraste con otros modelos de internacionalización como el paradigma ecléctico (Duning, 1988), las redes industriales y el grupo y ciclo de ventajas. En articulación con el modelo de redes, se plantea la importancia de las relaciones basadas en la confianza y en el conocimiento experiencial para consolidar la presencia exterior de la compañía.

Como crítica principal al modelo de Uppsala, se expone la discusión sobre su relevancia en los mercados contemporáneos (Johanson \& Vahlne, 1990), en especial porque las etapas que plantea son estructuradas en forma secuencial rígida, que difiere de procesos empresariales más acelerados y paralelos exigidos por el mercado contemporáneo. Además, se aborda el debate en referencia, al desconocimiento del mercado y el acceso a la información que son obstáculos del proceso de internacionalización según el modelo de Uppsala, pero donde el contexto global, permite la cercanía de tendencias de consumo y aspectos culturales, que, en conjunto con los medios de comunicación y la tecnología, facilitan la expansión de los negocios a mercados exteriores. Posteriormente, se realiza un contraste con el modelo de Paradigma Ecléctico, siendo ampliamente aceptado dentro los procesos de internacionalización, especialmente al análisis de la inversión extranjera (Guillén \& García-Canal, 2009).

La estrategia de internacionalización de la pequeña empresa latina, inicia sus procesos de comercialización externa en prácticas algunas veces complejas dadas las limitaciones de recursos de información y financieros, estando soportadas en estructuras comerciales austeras y simples, ofertando un portafolio caracterizado por una longitud corta en número de referencias, que compite con productos de mayor visibilidad en comercialización y rendimientos del país de origen (Johanson \& Vahlne, 1977).

Se identifica en la estrategia de internacionalización de la empresa la gradualidad en todo el proceso, toda vez que busca llegar los "mercados naturales" o cercanos, dada la ubicación geográfica cercana que favorece una mejor gestión logística de la distribución física internacional, lo cual incide poderosamente en las estructuras de costos de las firmas, siendo además relevante la identidad cultural, permitiendo la mejor adaptación del producto al nuevo mercado.

En el mismo sentido, la gradualidad se visualiza en la forma de ingreso paulatina a nuevos países, siendo preferente para las organizaciones la búsqueda de un distribuidor en el país destino, en lugar de realizar una operación directa por medio del establecimiento de fuerzas de ventas, oficinas, bodegas o plantas de producción, atenuando los riesgos de mercado y riesgos competitivos.

Estas relaciones de distribución las mantienen algunas empresas, dado el conocimiento institucional que posee el distribuidor en el país externo, pero también se identifican otras organizaciones que prefieren el manejo directo de la operación en el nuevo mercado, una vez se tenga conocimiento del mismo, a través del establecimiento de redes y formalización de convenios de distribución, maquila, y la adquisición de pequeños activos estratégicos. Una vez las firmas adquieren conocimiento 
y confianza en un mercado a través del fortalecimiento de la capacidad financiera, operativa, productiva y comercial, proceden a conquistar mercados más lejanos y sofisticados, incluso instalando pequeñas unidades productivas (Aulakh, Kotabe \& Teegen, 2000; Lopez et al., 2009).

Una característica común de las firmas latinoamericanas en el proceso de internacionalización, es la experiencia y conocimiento previo en su mercado interno, experiencia que le otorga habilidades básicas para enfrentar los mercados externos, no siendo ésta una forzosa regla, dado que algunas firmas inician su operación comercial a través de ventas eventuales a mercados externos. La caracterización anterior encaja en el modelo Uppsala, que se distingue por líneas de actuación enmarcadas en el previo desarrollo del mercado interno, una concepción gradualista de experiencia, compromiso y confianza, factores que permiten la creación de redes en la facilitación de un aprendizaje secuencial (Johanson \& Vahln, 2015; López et al., 2009).

Otra línea de estudio expuesta, es la que aborda el modelo de internacionalización a partir del enfoque springboard, el cual dirige su estrategia en la adquisición gradual de experiencia y conocimiento del mercado, articulado a la consecución de activos en el exterior y consolidación de alianzas empresariales (Luo \& Tung, 2007). Se describe a su vez que la estrategia springboard es útil para las multinacionales en el proceso de expansión a mercados emergentes, a través de la compra de activos estratégicos y la mitigación de ventajas competitivas en el país receptor (Aulakh et al., 2000).

Un elemento relevante entre los enfoques objeto de estudio, es la importancia de analizar los factores que permiten a las empresas de economías emergentes expandirse a operaciones internacionales. Planteando que las desventajas por la complejidad de sus mercados de origen se pueden convertir en ventajas para el desarrollo de mercados exteriores (Cuervo-Cazurra \& Genc, 2008).

En el mismo sentido, se expone que las multinacionales que provienen de países emergentes, debido a su experiencia al enfrentar un inestable entorno institucional y legal, en conjunto con complejas variables económicas y sociales en su país de origen; les permiten a estas empresas, desarrollar nuevas capacidades para competir en entornos desafiantes (Guillén \& García-Canal, 2009). También poseen una respuesta efectiva a los mercados a través de en una estrategia internacional que aprovecha el dinamismo de los mercados actuales.

En orientación a este fenómeno, se establece como referentes empresariales de internacionalización para latinoamérica, las empresas asiáticas, denominadas dragon multinationals que se han caracterizado por innovaciones estratégicas y organizacionales a partir de la expansión acelerada de sus economías emergentes, siendo identificadas como casos de éxito a nivel mundial (Mathews, 2006). Algunas de las empresas multinacionales de Asia, han sustentado su capacidad de llegar a nuevos mercados, a partir del aporte que realiza el enfoque institucional a la estrategia de los negocios internacionales (Peng, Wang \& Jiang, 2008; Wright, Filatotchev, Hoskisson \& Peng, 2005), en articulación con la relevancia que le han asignado al fortalecimiento de la industria y la gestión de recursos. 


\section{Documentos Estructurales (tronco)}

Los documentos estructurales son los que vinculan los recientes con los hegemónicos, su tasa de centralidad es la más alta, es decir, son citados de manera recurrente por las hojas y al mismo tiempo citan a las raíces, siendo por lo tanto los documentos que conectan la red. En la literatura consultada, se identifica principalmente la orientación de los procesos investigativos en tres enfoques: el desempeño de las empresas latinoamericanas en su apertura de mercados extranjeros, el análisis e identificación de factores que determinan su competencia en los mercados internacionales, y las condiciones determinantes del mercado emergente como país de origen en su proceso de internacionalización. Estos enfoques se abordan a partir de la relación con modelos teóricos inherentes a la estrategia empresarial, y el desarrollo de mercado. Además, se presenta en múltiples artículos, la evaluación de ciertas variables a través de estudios empíricos con fuentes primarias representadas por empresas latinoamericanas que han desarrollado procesos de apertura de mercados.

En el primer enfoque, sobre el desempeño empresarial, se aborda la relación entre dicho desempeño y el proceso de internacionalización, donde se aduce las múltiples ventajas que ofrece la apertura de mercados a las compañías en sus etapas paulatinas de crecimiento y expansión. Sin embargo, se plantea un camino de relación negativo-positivo-negativo en cuanto a rendimiento financiero se refiere, ya que en la primera etapa de apertura a nuevos mercados los costos incrementales exceden los beneficios, para posteriormente en la segunda etapa el efecto de una mayor expansión multinacional genera rendimientos positivos, y finalmente en una tercera etapa, una expansión internacional excesiva, genera que los costos incrementales superen los beneficios (Vélez-Calle, Sanchez-Henriquez \& Contractor, 2018). En relación a este enfoque, también se plantea que la diversificación de mercados e internacionalización de la empresa se conciben como un factor para mejorar su desempeño organizacional, exponiendo que existen límites para los efectos positivos que le brinda el mercado exterior, y donde se percibe una relación más fuerte en cuanto a mejora del desempeño para las empresas de servicios que para las manufactureras (Borda, Geleilate, Newburry \& Kundu, 2017).

En relación al enfoque que comprende los factores determinantes para competir en el mercado internacional, se abordan estudios sobre la importancia de la ubicación geográfica, en cuanto a la cercanía con mercados potenciales, la geoeconomía, el acceso a recursos y las alianzas estratégicas públicas y privadas (Conti, Parente, \& de Vasconcelos, 2016; Dau, 2013; Felzensztein, Ciravegna, Robson \& Amorós, 2015; Vendrell-Herrero, Gomes, Mellahi \& Child, 2017). Se presenta una visión general de los cambios del entorno empresarial en América Latina y su evolución en los procesos de internacionalización (Carneiro \& Brenes, 2014) (Hennart, Sheng \& Carrera, 2017) (Ciravegna, López \& Kundu, 2016). También se estudia la importancia de expandir negocios a nuevos países, a partir de la generación de valor agregado, aumento de subsidiarias y canales comerciales, y la adopción del paradigma ecléctico (CuervoCazurra, 2007), en conjunto con el aprovechamiento de las reformas pro-mercado que reducen las asimetrías institucionales, aumentan el flujo comercial y favorecen 
la competencia, obligando a las empresas a expandirse a segmentos internacionales (Dau, 2012).

Finalmente, se identifica el enfoque de estudio que comprende las condiciones del entorno empresarial de los países emergentes, como una realidad que incide directamente en el modelo a través del cual las compañías se atreven a incursionar en los negocios internacionales (Cuervo-Cazurra, Ciravegna, Melgarejo \& López, 2018). Las fuentes consultadas plantean la importante relevancia e incidencia de las condiciones complejas e inestables de los países en vía de desarrollo en América Latina, en las estrategias empresariales de internacionalización, donde la gestión institucional (Brenes, Ciravegna \& Pichardo, 2019), las condiciones económicas (Aguilera et al., 2017), la inversión extranjera directa (Cuervo-Cazurra, 2008), y la atracción de talento humano calificado (Newburry, Gardberg \& Sánchez, 2014), en conjunto con la intensidad competitiva (Martin \& Javalgi, 2016), se convierten en factores determinantes para las empresas latinoamericanas, que una vez superados y con la experiencia y fortaleza adquirida, les permite introducirse en el campo de los negocios internacionales.

\section{Documentos recientes (hojas)}

Los artículos con el indicador más alto de salida son los que involucran como referentes los demás documentos del árbol, es decir, raíces y tronco, a estos se les asigna la categoría de hojas. En esta categoría, se encuentra que los documentos analizados plantean que la estrategia de internacionalización depende de muchos factores internos y externos de las firmas como es su tamaño, las habilidades gerenciales y variables latentes (Botero-Pinzón, Casillas \& Valencia-Cárdenas, 2019), además su propiedad familiar, número de socios, ubicación geográfica, grado de desarrollo tecnológico (Bianchi, Glavas \& Mathews, 2017), y sector económico en que opera la empresa, ya que definen las condiciones para acceder a nuevos mercados, en articulación con el contexto del país destino, su grado de desarrollo, aspectos institucionales, de mercado, estabilidad jurídica, entre otros aspectos a considerar.

Se evidencia que el modelo Uppsala (Johanson \& Vahlne, 1977) es aplicado por muchas pymes de países emergentes, pero no se puede establecer como una generalidad, dada las características propias de las empresas en sus aspectos internos como se señaló anteriormente, sino también en las condiciones de los mercados a los cuales las empresas quieren llegar (Malhotra, Lin \& Farrell, 2016); razón por la cual en la revisión bibliográfica se obtiene, que la estrategia de internacionalización se adquiere características multidimensionales en su evaluación, debido a la cantidad de variables de diverso tipo que inciden en el diseño de dicha estrategia.

Las variables latentes, además de los recursos y capacidades organizacionales (Bianchi, Carneiro \& Wickramasekera, 2018), las capacidades tecnológicas desarrolladas en campos como marketing, finanzas, recursos humanos y relaciones internacionales, adquieren relevancia especial (Carneiro, Bamiatzi \& Cavusgil, 2018; Malhotra et al., 2016), entendiendo que soportan la habilidad para gestionar la operación entre el país de origen y los mercados destino. En la concepción de los recursos 
y capacidades de la firma, estas competencias organizacionales le facilitan superar limitaciones de experiencia como factor moderador (Batsakis \& Mohr, 2017), así como el desconocimiento internacional de las operaciones inherentes a la actividad comercial y la superación de barreras ambientales externas, como también la velocidad de diversificación geográfica y de portafolio, en conjunto con la gestión eficiente en costos de transacción involucrados en el ejercicio de expansión a otras naciones (Batsakis \& Mohr, 2017).

Los beneficios operativos asociados a factores como la regionalización, se evidencian en empresas de tecnología y farmacéutica, teniendo a la India como ejemplo de país emergente en sus proceso de expansión a los mercados Norteamericano, Europeo, Latinoamericano (Batsakis \& Mohr, 2017; Varma, Bhasin \& Nayyar, 2015), y en regiones del oriente a China y Japón, en la vque el factor institucional coadyuva en los procesos de internacionalización (Batsakis \& Mohr, 2017; De Resende, Borini \& Ogasavara, 2019; Varma et al., 2015), y donde el tamaño de la competencia y del mercado, influyen en la toma de decisiones de inversión extranjera directa-IED. Contrario a estrategias de empresas multinacionales chinas que aprovechan la debilidad institucional de países emergentes para favorecer su ingreso, en especial en industrias extractivas (Lin, 2015).

De otra parte, la incertidumbre internacional juega un papel fundamental en las intenciones de internacionalización de las firmas (Malhotra et al., 2016), reforzada por las percepciones de distancia geográfica, apreciación cultural y obviamente los factores institucionales propicios para el desarrollo del plan de extensión a otros mercados. Aspectos relativos a estabilidad jurídica y de cumplimiento de contratos (De Resende et al., 2019), la evaluación de riesgo país y de soberanía, sofisticación del mercado, la libertad comercial, facilidad de expatriación de utilidades; todas hacen parte de las variables que evalúan empresas multinacionales de países desarrollados (Huesca-Dorantes, Michailova \& Stringer, 2018), y las multilatinas de países emergentes, especialmente de aquellos con mejor desempeño internacional como México, Chile, Brasil y Argentina.

Es importante señalar que la literatura explorada, menciona que las empresas familiares tienen mayores posibilidades de ingreso a mercados externos y obtener un desempeño importante en sus exportaciones, en la medida que posean juntas directivas fuertes (Huesca-Dorantes et al., 2018), con experiencia y audacia, en una razonable aversión al riesgo y la gestión eficiente que atenúe los problemas de agencia. Lo anterior es corroborado por la experiencia de las multilatinas mexicanas, que además de los lazos familiares (Huesca-Dorantes et al., 2018), se ven beneficiadas en los vínculos con el gobierno, afiliaciones políticas y por grupos de países cercanos, implementando como estrategia la compra de activos estratégicos en dichos países (Huesca-Dorantes et al., 2018; Omokaro-Romanus, Anchor \& Konara, 2019), acudiendo además a las fusiones y adquisiciones.

La revisión bibliográfica, evidencia los resultados de estudios que expresan la importancia de la holgura organizacional de firmas que proporcionan facilidades en los procesos de internacionalización (Carneiro et al., 2018), al reforzarse la condición de las variables latentes antes mencionadas, además de los consabidos factores 
institucionales, condiciones de experiencia y confianza lograda en los mercados. Lo anterior independiente de la manera en la cual surge el proceso de internacionalización, bien sea porque la firma pudo desarrollar una experiencia interna, o que provee después una trayectoria internacional temprana, a diferencia de firmas que su ingreso a mercados externos es un resultado inesperado o fortuito, o de manera contraria, es producto de un modo emprendedor o de una estrategia planificada (Wright et al., 2005).

\section{Perspectivas}

A partir de la revisión de los documentos involucrados, se identificaron a través del algoritmo de clusterización 4 perspectivas o líneas principales de investigación, que de manera conjunta agrupan más del 64\% (460 artículos) de la red (Figura 6), a continuación, son presentadas y analizadas:

\begin{tabular}{|c|c|}
\hline $\begin{array}{c}\text { Perspective } 1 \\
\text { Diversificación y rendimiento } \\
17 \% \text { of network } \\
118 \text { Nodos } \\
195 \text { conexiones }\end{array}$ & $\begin{array}{c}\text { Perspective } 2 \\
\text { Emprendimiento y redes } \\
\text { 17\% of network } \\
118 \text { Nodos } \\
197 \text { conexiones }\end{array}$ \\
\hline 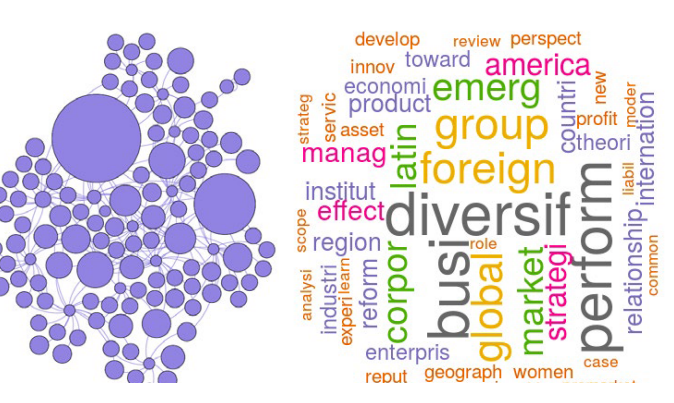 & 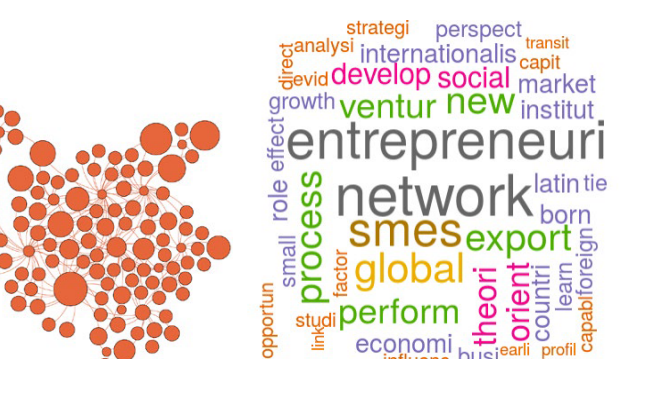 \\
\hline $\begin{array}{c}\text { Perspectiva } 3 \\
\text { Exportaciones y mercados } \\
15 \% \text { of network } \\
108 \text { Nodos } \\
203 \text { Conexiones }\end{array}$ & $\begin{array}{c}\text { Perspectiva } 4 \\
\text { Estrategias y competenci } \\
15 \% \text { of network } \\
116 \text { Nodos } \\
187 \text { Conexiones }\end{array}$ \\
\hline 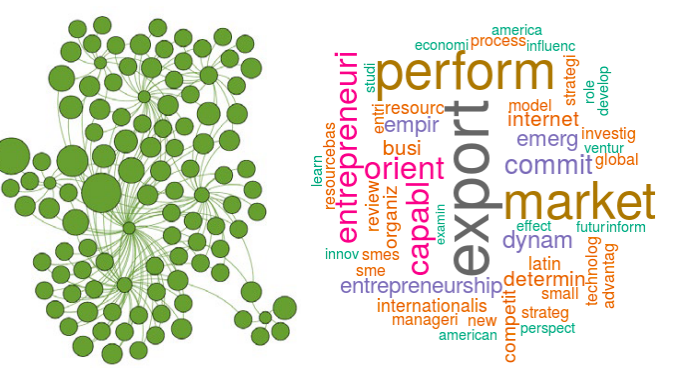 & 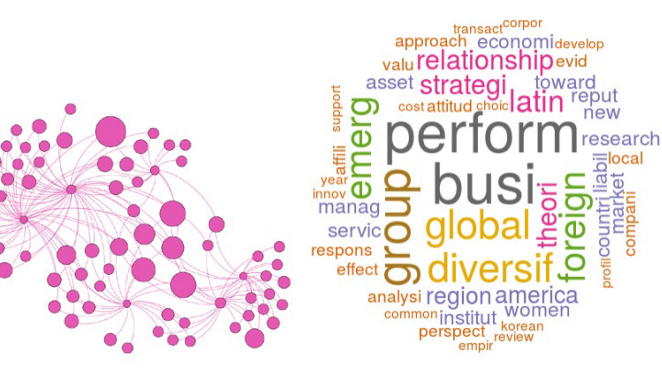 \\
\hline
\end{tabular}

Figura 6. Perspectiva

Fuente: Elaboración propia. 


\section{Perspectiva 1: Diversificación y Desempeño}

La revisión literaria evidencia que la estrategia de internacionalización se soporta en diferentes variables de evaluación interna de la organización y país de origen de la firma, y de factores externos del mercado destino. Las estrategias de internacionalización de acceso se establecen entre la concentración y la diversificación, siendo la concentración una estrategia que exige un producto de mayor diferenciación y personalización, buscando una importante aprobación del producto, exigiendo una eficiencia en la estructura de costes derivado de la exigencia de una holgura gerencial, específicamente de variables latentes como relaciones internacionales, marketing, finanzas, recurso humano y tecnología (Botero-Pinzón et al., 2019). Esta estrategia busca centrar la operación internacional en países específicos, que aparte de los fines comerciales, se esfuerza en disminuir costos logísticos y administrativos que subyacen del arribo a mercados externos (Batsakis \& Mohr, 2017; Botero-Pinzón et al., 2019).

La estrategia de diversificación puede orientarse al portafolio, ampliando y profundizando la propuesta comercial de bienes y servicios; la expansión a países destino, bajo el modelo Uppsala o una estrategia más dinámica, a través de la adaptación productos al perfil de cada país, o bien la combinación de las dos anteriores, que supone un músculo financiero, operativo, tecnológico y comercial de mayor envergadura para la firma. Lo anterior le concede a la empresa la posibilidad de disminución del grado de riesgos (Batsakis \& Mohr, 2017; Botero-Pinzón et al., 2019; De Resende et al., 2019), en donde los resultados de su actividad internacional tiene la posibilidad de valorarse en una mercado más amplio. De todas formas esta estrategia posee variables que afectan su desempeño como situación de riesgo país, estabilidad institucional y jurídica según Carneiro et al.(2018), destacando que se debe implementar una estrategia diferencial de acuerdo al segmento de mercado a incursionar (Carneiro et al., 2018; Lin, 2015).

Finalmente en esta perspectiva, se induce la necesidad de establecer nuevas líneas de investigación que relacionen la diversificación con procesos de apoyo institucional de manera estructurada, y de programas de emprendimiento por parte de los gobiernos del país originario de la firma; asimismo en aspectos como acceso a crédito, estímulos tributarios, generación de habilidades para la internacionalización y su acompañamiento, en conjunto con estudios del rol de la triple hélice estado-empresauniversidad en los desempeños de la internacionalización de la pyme de mercados emergentes.

\section{Perspectiva 2: Emprendimiento y Redes}

En el compendio del material bibliográfico de esta perspectiva, se abordan los diferentes modelos y factores de internacionalización presentados en pymes y unidades de emprendimiento, las cuales en etapas tempranas incursionan en mercados exteriores a un ritmo más acelerado; a partir de estudios con fundamentación teórica relacionada con procesos de internacionalización en articulación con casos de estudios 
con empresas principalmente de Brasil (De Farias, Nataraajan \& Kovacs, 2009; Da Rocha, Cotta, Pacheco \& De Abreu, 2012), México (Fernández-Moya, 2012; Larios, 2018; Martin \& Javalgi, 2016) y Chile (Felzensztein et al., 2015).

Se identifica como uno de los objetos de estudio más abordados, el rápido dinamismo que tienen las pequeñas empresas y los modelos de emprendimiento actuales para acceder a mercados internacionales, denominadas como born global (Bell, McNaughton, Young \& Crick, 2003). Se destacan como dos de los factores que han favorecido los procesos de internacionalización de las empresas latinoamericanas, las características de ser países con economías emergentes y la liberalización de sus mercados (Domínguez \& Brenes, 1997). Respecto al factor que comprende las economías emergentes, se plantea como una situación que establece adversidades locales e inestabilidad institucional en el país de origen pero a su vez se convierte en un catalizador de la necesidad por identificar y aprovechar nuevas oportunidades internacionales, donde el emprendimiento social se convierte en una de las líneas potenciales (Chen, Saarenketo \& Puumalainen, 2016). En el estudio que aborda las oportunidades internacionales, se explica que el contexto, la dinámica y la variedad son categorías las cuales permiten a las empresas realizar procesos tempranos de apertura mercados globales (Reuber, Dimitratos \& Kuivalainen, 2017).

La literatura explorada evidencia que la orientación empresarial, las redes industriales y de conocimiento (Ciravegna, López \& Kundu, 2014; Felzensztein et al., 2015; Fernández-Moya, 2012; Martin \& Javalgi, 2016), y la inversión extranjera (Da Rocha et al., 2012), son canales que han incidido favorablemente en los procesos de internacionalización de pymes y unidades de emprendimiento latinoamericanas en su etapas tempranas de expansión; ya que han acelerado y facilitado su incursión en nuevos mercados a través del acceso a recursos, aliados estratégicos e información.

Se identificaron como principales líneas investigativas a explorar en posteriores estudios, el análisis de modelos empíricos de internacionalización de unidades de negocio en sus etapas tempranas de emprendimiento, con el fin de identificar aquellos factores que han acelerado y facilitado la expansión a mercados exteriores de las pymes latinoamericanas, convirtiéndose en un tema aún por desarrollar y abordar a partir de fundamentos teóricos y conceptos propios de la estrategia de negocios.

\section{Perspectiva 3: Exportaciones y Mercado}

El trabajo bibliométrico desarrollado en este documento, permitió establecer que la relación entre la actividad exportadora de las firmas, en especial de las pymes y su desempeño en la aplicación de la estrategia de internacionalización, depende de muchos factores internos y externos, desde la perspectiva de habilidades generadas en combinación intencional de recursos para llegar a otros mercados. Es así como variables claves como el conocimiento, la gradualidad (Johanson \& Vahlne, 2015), la experiencia, la actitud emprendedora, los recursos, y capacidades (especialmente los intangibles) que generan habilidades (Bianchi et al., 2018), han contribuido a resultados exitosos de pymes de mercados emergentes latinoamericanos. 
El impacto de la internacionalización de la firma tiene una relación directa con el desempeño de las exportaciones (Aguilera et al., 2017), por lo cual de la revisión bibliográfica se puede infereir que de una parte del éxito de la estrategia se encuentra soportada en la gradualidad y de otros procesos de emprendimiento acelerado, muchas veces sujetos al azar de los mercados destino (Ciravegna, Kundu, Kuivalainen \& López, 2019), lo que implica incurrir en altos costos de transacción dadas unas condiciones las cuales exigen una velocidad de diversificación de mercados geográficos asimilables y cercanos; $y$ de un portafolio sustentado en productos internacionalmente adaptables a a una demanda específica y perfiles de consumo que subyacen de identidad cultural (Batsakis \& Mohr, 2017).

En contraste con las empresas multinacionales latinas cuyo propiedad está dada por fuertes ascendencias familiares que dinamizan su ejercicio exportador desde la penetración plena del mercado interno y la expansión a mercados emergentes latinoamericanos aprovechando la adquisición de activos estratégicos los cuales traen consigo acceso a base de clientes, proveedores y distribuidores, además de recursos intangibles del recurso humano que se adhiere a la nueva organización (Herrera-Echeverri, Geleilate, Gaitan-Riaño, Haar \& Soto-Echeverry, 2016).

Es preciso destacar que las firmas deben evaluar la diferencia de ingresar a mercados de naciones emergentes a diferencia de mercados de países desarrollados, en los cuales no solo el marco institucional determina un mayor compromiso financiero (Malhotra et al., 2016), que resultan en la exigencia de una mayor capacidad y holgura gerencial (Mathews, 2006) para avanzar en la incursión del mercado exterior y el exhaustivo análisis de los productos o servicios a exportar, advirtiendo además las necesidades de control en la gestión de la distribución en el mercado al cual se ingresa, especialmente en las fases iniciales del proceso de internacionalización, en donde se prefiere el manejo de una gradualidad en su forma de ingreso (Johanson \& Vahlne, 1977), buscando adquirir aprendizajes y adherir el conocimiento institucional del mercado que tiene el distribuidor como una capacidad estratégica (Bianchi et al., 2018).

Esta perspectiva ilustra la necesidad de fortalecer a futuro el ejercicio investigativo de la internacionalización de la firma, teniendo en cuenta la consideración de recursos y capacidades y su uso intencional para la consecución de habilidades y competencias en los procesos de exportación, dadas unas restricciones de capital y condiciones institucionales de los países de origen de estas compañías.

\section{Perspectiva 4: Estrategias y Competencia}

Entre el conjunto de las publicaciones bibliográficas abordadas, se destaca una perspectiva orientada a analizar la importancia de la estrategia y la diversificación, en el proceso de internacionalización de empresas latinoamericanas o de países emergentes. Donde factores como la capacidad para profundizar y ampliar el alcance en los mercados internacionales, se convierte en una estrategia que incide en el rendimiento financiero de las empresas latinoamericanas (Vélez-Calle et al., 2018); además se plantea a partir de un estudio empírico en compañías multinacionales brasileñas, que el proceso de internacionalización también depende de la capacidad y estrategia 
de innovación de la empresa (Fleury, Leme \& Mendes, 2013); También se aborda la incidencia de los costos de transacción en la estrategia para acceder a mercados exteriores, concluyendo que es más fácil desarrollar mercados regionales que mercados globales dado las restricciones que generan dichos costos de transacción (Rugman \& Verbeke, 2004b).

Se abordaron estudios donde se realiza una evaluación comparativa entre empresas internacionales que provienen de un país emergente versus las empresas que provienen de un país desarrollado, estableciendo que existen ventajas estratégicas no desarrolladas directamente con el mercado, más bien, basadas en la teoría de recursos y el concepto de distancia (Cuervo-Cazurra \& Genc, 2011; Rugman \& Verbeke, 2004a). En Orientación a este enfoque, los países latinoamericanos emergentes han desarrollado políticas para darle apertura a sus mercados internos, a la inversión extranjera, y a la competencia internacional, reduciendo la intervención del gobierno e incentivando el comercio regional; lo cual ha permitido el surgimiento de compañías orientadas al mercado internacional con estrategias que le permitan enfrentar este contexto (Brenes \& Domínguez, 1997).

En esta perspectiva se relacionan múltiples estudios la relevancia de la diversificación en la estrategia de las compañías latinoamericanas con presencia en mercados internacionales, donde se orienta en la ampliación de los segmentos de mercados, su participación transfronteriza y la expansión de sus filiales comerciales presentando una relación directa con el rendimiento financiero de la compañía y diferenciando su influencia a partir de su naturaleza de servicios o manufacturera (Borda et al., 2017; Capar \& Kotabe, 2003; Wiersema \& Bowen, 2011).

Como líneas futuras por investigar, propuestas a partir de las fuentes bibliográficas de esta perspectiva, se identifica la necesidad de estudiar las estrategias específicas y tipos de diversificación que están desarrollando las empresas latinoamericanas, para ser más competitivas en los mercados internacionales.

\section{CONCLUSIONES Y LIMITACIONES}

Se observa un aumento del interés por parte de la comunidad científica entorno a la investigación en internacionalización de empresas latinas, esto se evidencia a través del incremento de las publicaciones sobre el tema en los últimos años, la cual reporta una tasa de crecimiento anual del 18\%, acelerándose de forma importante en los últimos 5 años. Luciano Ciravegna es el autor con mayor número de publicaciones relacionadas al tema, sin embargo, Alvaro Cuervo Cazurra es el autor más importante en la red de cocitaciones, (en términos bibliométricos), además cuenta con el mayor número de conexiones y referencias. La producción científica relacionada al tema se origina en su mayoría en Estados Unidos y España, sin embargo, el primer país Latinoamericano en aparecer en el top 10 es Colombia, ocupando la tercera posición en el listado general.

Se identifica como un planteamiento en el que converge el objeto de estudio de múltiples publicaciones bibliográficas abordadas, la relación dada entre los procesos de internacionalización de empresas latinoamericanas y el contexto de su país de 
origen como mercado emergente. Se evidencia que están estrechamente articuladas sus estrategias de negocios internacionales con las condiciones locales de inestabilidad económica, política, social e institucional, ya que las compañías que logran superar estas limitantes internas, presentan factores de competitividad que pueden considerarse dinamizadores un contexto internacional.

Un fenómeno contemporáneo estudiado en la literatura científica, es el Born Global y el emprendimiento, especialmente por la facilidad con la que unidades de negocio actuales acceden a los mercados exteriores y desarrollan procesos de internacionalización en etapas tempranas de constitución organizacional. Presentando por lo tanto un contraste con modelos clásicos de gradualidad y expansión paulatina hacia segmentos exteriores, dado que las pymes modernas aceleran su dinamicas de apertura extranjera impulsadas en factores catalizadores como las redes de conocimiento, redes industriales, la diversificación comercial, el libre comercio, la inversión extranjera y los avances tecnológicos que acercan los mercados.

Se resalta que el modelo de Uppsala es el punto de partida clásico para el análisis de la producción científica relacionada, estableciendo en conjunto que existen comprobados factores internos, que son determinantes en la obtención de logros empresariales en el proceso de internacionalización. Las diferentes teorías de internacionalización de las pymes encuentran un punto de convergencia como es la generación de redes empresariales (Johanson \& Vahlne, 1990), la cual se da como el resultado de las interacciones entre las empresas permanentes de las firmas locales con las redes del exterior, consolidando canales para la internacionalización de las empresas a partir del sistema en el que interactúa y afianza alianzas estratégicas, que le permitan acceder a nuevos mercados extranjeros. También debe considerarse el tamaño de la empresa y subyacente de esta situación, está la suficiencia u holgura organizacional (Carneiro et al., 2018), en aspectos financieros, administrativos, operativos, comerciales, entre otros a considerar, que le conceden la adquisición de recursos y capacidades (Bianchi et al., 2018), como unos de los factores de trascendencia organizacional, dado que le permite superar la falta de experiencia y desconocimiento del ejercicio operativo y comercial en la internacionalización de la empresa.

A pesar de que existen empresas latinoamericanas con una larga y reconocida trayectoria a nivel local y regional, solo cuando realizan procesos de internacionalización a mercados importantes como el de Estados Unidos son realmente visibles y adquieren un mayor protagonismo, debiendo superar barreras de todo tipo para obtener ese logro (Aguilera et al., 2017).

\section{Futuras Líneas de Investigación}

Las perspectivas para futuras investigaciones son numerosas, en especial aquellas que relacionen las restricciones de capital, condiciones institucionales precarias del país de origen de la firma y casos exitosos en proceso de internacionalización dadas unas condiciones especiales de recursos y capacidades, específicamente en aquellos intangibles. Así mismo se vislumbran caminos investigativos en la correspondencia de internacionalización y los efectos resultantes de la alianza universidad-empresa- 
estado, además del resultado de la gobernanza y la responsabilidad social empresarial en la construcción de reputación y la incidencia de la internacionalización de la firma.

También se requiere orientar las investigaciones en el estudio y determinación de los factores que contribuyen al desarrollo de procesos de internacionalización en los modelos de emprendimiento latinoamericanos en sus etapas tempranas, en conjunto con la identificación de estrategias de mercado específicas y tipos de diversificación empleados para acceder a nuevos mercados exteriores.

\section{REFERENCIAS}

Aguilera, R. V., Ciravegna, L., Cuervo-Cazurra, A. \& Gonzalez-Pérez, M. A. (2017). Multilatinas and the internationalization of Latin American firms. Journal of World Business, 52(4), 447-460. https://doi.org/10.1016/j.jwb.2017.05.006

Aria, M. \& Cuccurullo, C. (2017). bibliometrix: An R-tool for comprehensive science mapping analysis. Journal of Informetrics, 11(4), 959-975. https://doi. org/10.1016/j.joi.2017.08.007

Aulakh, P. S., Kotabe, M. \& Teegen, H. (2000). Export strategies and performance of firms from emerging economies: evidence from Brazil, Chile, and Mexico. Academy of Management Journal, 43(3), 342-361. Available: https://www.jstor.org/ stable/1556399

Batsakis, G. \& Mohr, A. T. (2017). Revisiting the relationship between product diversification and internationalization process in the context of emerging market MNEs. Journal of World Business, 52(4), 564-577. https://doi.org/10.1016/j. jwb.2016.11.005

Bell, J., McNaughton, R., Young, S. \& Crick, D. (2003). Towards an Integrative Model of Small Firm Internationalisation. Journal of International Entrepreneurship, 1(4), 339-362. https://doi.org/10.1023/A:1025629424041

Bianchi, C., Carneiro, J. \& Wickramasekera, R. (2018). Internationalisation commitment of emerging market firms. Journal of Small Business and Enterprise Development, 25(2), 201-221. https://doi.org/10.1108/jsbed-07-2017-0221

Bianchi, C., Glavas, C. \& Mathews, S. (2017). SME international performance in Latin America. Journal of Small Business and Enterprise Development, 24(1), 176-195. https://doi.org/10.1108/jsbed-09-2016-0142

Blondel, V. D., Guillaume, J.-L., Lambiotte, R. \& Lefebvre, E. (2008). Fast unfolding of communities in large networks. Journal of Statistical Mechanics: Theory and Experiment, (10), 1-12. https://doi.org/10.1088/1742-5468/2008/10/p10008

Borda, A., Geleilate, J.-M. G., Newburry, W., \& Kundu, S. K. (2017). Firm internationalization, business group diversification and firm performance: The case of Latin American firms. Journal of Business Research, 72, 104-113. https://doi. org/10.1016/j.jbusres.2016.11.006

Borda, A., Newburry, W., Teegen, H., Montero, A., Nájera-Sánchez, J. J., Forcadell, F., Lama, N. \& Quispe, Z. (2017). Looking for a service opening: Building reputation by leveraging international activities and host country context. Journal of World Business, 52(4), 503-517. https://doi.org/10.1016/j.jwb.2016.08.006 
Botero-Pinzón, L.-D., Casillas, J. C. \& Valencia-Cárdenas, M. (2019). The international profile of the firm: Application of latent profile analysis to the case of Colombia. Academia Revista Latinoamericana de Administración, 32(3), 392410. https://doi.org/10.1108/ARLA-04-2018-0084

Brenes, E. R., Ciravegna, L. \& Pichardo, C. A. (2019). Managing institutional voids: A configurational approach to understanding high performance antecedents. Journal of Business Research, 105(C), 345-358. https://doi.org/10.1016/j. jbusres.2018.03.022

Brenes, E. R. \& Domínguez, L. V. (1997). Prologue to this special issue: Strategic choices in the new international enterprise in Latin America. Journal of Business Research, 38(1), 1-2. https://doi.org/10.1016/S0148-2963(96)00112-9

Buitrago, S., Duque, P. \& Robledo, S. (2020). Branding Corporativo: una revisión bibliográfica. Económicas CUC, 41(1), 143-162. https://doi.org/10.17981/econcuc.41.1.2020.Org.1

Camargo, D., Cardona, O. \& Mira, G. (2017). Institucionalismo económico e internacionalización de empresas: una revisión de literatura. Económicas CUC, 38(1), 185-206. https://doi.org/10.17981/econcuc.38.1.09

Capar, N. \& Kotabe, M. (2003). The relationship between international diversification and performance in service firms. Journal of International Business Studies, 34(4), 345-355. https://doi.org/10.1057/palgrave.jibs. 8400036

Carneiro, J. \& Brenes, E. R. (2014). Latin American firms competing in the global economy. Journal of Business Research, 67(5), 831-836. https://oi.org/10.1016/j. jbusres.2013.07.001

Carneiro, J., Bamiatzi, V. \& Cavusgil, S. T. (2018). Organizational slack as an enabler of internationalization: The case of large Brazilian firms. International Business Review, 27(5), 1057-1064. https://doi.org/10.1016/j.ibusrev.2018.03.006

Chen, J., Saarenketo, S. \& Puumalainen, K. (2016). Internationalization and value orientation of entrepreneurial ventures - a Latin American perspective. Journal of International Entrepreneurship, 14(1), 32-51. https://doi.org/10.1007/ s10843-016-0169-9

Ciravegna, L., Kundu, S. K., Kuivalainen, O. \& López, L. E. (2019). The timing of internationalization - Drivers and outcomes. Journal of Business Research, 105(C), 322-332. https://doi.org/10.1016/j.jbusres.2018.08.006

Ciravegna, L., López, L. E. \& Kundu, S. K. (2016). The internationalization of Latin American enterprises-Empirical and theoretical perspectives. Journal of Business Research, 69(6), 1957-1962. https://doi.org/10.1016/j.jbusres.2015.10.141

Ciravegna, L., López, L. \& Kundu, S. (2014). Country of origin and network effects on internationalization: A comparative study of SMEs from an emerging and developed economy. Journal of Business Research, 67(5), 916-923. https://doi. org/10.1016/j.jbusres.2013.07.011

Conti, C. R., Parente, R. \& de Vasconcelos, F. C. (2016). When distance does not matter: Implications for Latin American multinationals. Journal of Business Research, 69(6), 1980-1992. https://doi.org/10.1016/j.jbusres.2015.10.144 
Cuervo-Cazurra, A. (2008). The multinationalization of developing country MNEs: The case of multilatinas. Journal of International Management, 14(2), 138-154. https://doi.org/10.1016/j.intman.2007.09.001

Cuervo-Cazurra, A. (2007). Sequence of value-added activities in the multinationalization of developing country firms. Journal of International Management, 13(3), 258-277. https://doi.org/10.1016/j.intman.2007.05.009

Cuervo-Cazurra, A. \& Genc, M. E. (2011). Obligating, Pressuring, and Supporting Dimensions of the Environment and the Non-Market Advantages of Developing/ Country Multinational Companies. Journal of Management Studies, 48(2), 441455. https://doi.org/10.1111/j.1467-6486.2010.00964.x

Cuervo-Cazurra, A. \& Genc, M. (2008). Transforming disadvantages into advantages: developing-country MNEs in the least developed countries. Journal of International Business Studies, 39(6), 957-979. https://doi.org/10.1057/palgrave. jibs. 8400390

Cuervo-Cazurra, A. \& Dau, L. A. (2009). Promarket Reforms and Firm profitability in Developing Countries. Academy of Management Journal, 52(6), 1348-1368. https://doi.org/10.5465/amj.2009.47085192

Cuervo-Cazurra, A., Ciravegna, L., Melgarejo, M. \& López, L. (2018). Home country uncertainty and the internationalization-performance relationship: Building an uncertainty management capability. Journal of World Business, 53(2), 209-221. https://doi.org/10.1016/j.jwb.2017.11.002

Da Rocha, A. Cotta, R., Pacheco, H. \& De Abreu, I. (2012). The international commitment of late-internationalizing Brazilian entrepreneurial firms. International Marketing Review, 29(3), 228-252. https://doi.org/10.1108/02651331211229741

Dau, L. A. (2013). Learning across geographic space: Pro-market reforms, multinationalization strategy, and profitability. Journal of International Business Studies, 44(3), 235-262. https://doi.org/10.1057/jibs.2013.5

Dau, L. A. (2012). Pro-market reforms and developing country multinational corporations: Reforms and DMNCs. Global Strategy Journal, 2(3), 262-276. https:// doi.org/10.1111/j.2042-5805.2012.01033.x

De Farias, S. A., Nataraajan, R. \& Kovacs, E. P. (2009). Global business partnering among family-owned enterprises. Journal of Business Research, 62(6), 667-672. https://doi.org/10.1016/j.jbusres.2008.02.003

De Resende, P. L., Borini, F. M. \& Ogasavara, M. H. (2019). Latin American franchise internationalization: The impact of institutional environment. Thunderbird International Business Review, 61(2), 217-228. https://oi.org/10.1002/tie.21975

Domínguez, L. V. \& Brenes, E. R. (1997). The internationalization of Latin American enterprises and market liberalization in the Americas: A vital linkage. Journal of Business Research, 38(1), 3-16. https://doi.org/10.1016/S0148-2963(96)001130

Dunning, J. H. (1988). The Eclectic Paradigm of International Production: A Restatement and Some Possible Extensions. In: Cantwell J. (eds), The Eclectic Paradigm (pp. 50-84). London: Palgrave Macmillan. https://doi.org/10.1007/978-1137-54471-1_3 
Duque, P. \& Cervantes-Cervantes, L.-S. (2019). Responsabilidad Social Universitaria: una revisión sistemática y análisis bibliométrico. Estudios Gerenciales, 35(153), 451-464. https://doi.org/10.18046/j.estger.2019.153.3389

Felzensztein, C., Ciravegna, L., Robson, P. \& Amorós, J. E. (2015). Networks, Entrepreneurial Orientation, and Internationalization Scope: Evidence from Chilean Small and Medium Enterprises. Journal of Small Business Management, 53(1), 145-160. https://doi.org/10.1111/jsbm.12188

Ferguson, R. (2012). Learning analytics: drivers, developments and challenges. International Journal of Technology Enhanced Learning, 4(5/6), 304-317. https:// doi.org/10.1504/IJTEL.2012.051816

Fernández-Moya, M. (2012). Creating Knowledge Networks: Spanish Multinational Publishers in Mexico. Business History Review, 86(1), 69-98. https://doi. org/10.1017/S0007680512000049

Fleury, A., Leme, M. T. \& Mendes, F. (2013). The Brazilian Multinationals' Approaches to Innovation. Journal of International Management, 19(3), 260-275. https://doi.org/10.1016/j.intman.2013.03.003

Freeman, L. C. (1977). A Set of Measures of Centrality Based on Betweenness. Sociometry, 40(1), 35-41 . https://doi.org/10.2307/3033543

Guillén, M. F. \& García-Canal, E. (2009). The American Model of the Multinational Firm and the "New" Multinationals From Emerging Economies. Academy of Management Perspectives, 23(2), 23-35. https://doi.org/10.5465/amp.2009.39985538

Hennart, J.-F., Sheng, H. H. \& Carrera, J. M. (2017). Openness, international champions, and the internationalization of Multilatinas. Journal of World Business, 52(4), 518-532. https://doi.org/10.1016/j.jwb.2016.08.005

Herrera-Echeverri, H., Geleilate, J. G., Gaitan-Riaño, S., Haar, J. \& Soto-Echeverry, N. (2016). Export behavior and board independence in Colombian family firms: The reverse causality relationship. Journal of Business Research, 69(6), 20182029. https://doi.org/10.1016/j.jbusres.2015.10.147

Hirsch, J. E. (2005). An index to quantify an individual's scientific research output. Proceedings of the National Academy of Sciences of the United States of America, 102(46), 16569-16572. https://doi.org/10.1073/pnas.0507655102

Huesca-Dorantes, J. L., Michailova, S. \& Stringer, C. (2018). Aztec multilatinas: characteristics and strategies of Mexican multinationals. Review of International Business and Strategy, 28(1), 2-18. https://oi.org/10.1108/ribs-06-2017-0046

Indiana University and SciTech Strategies. (2009). Sci2 Tool: A Tool for Science of Science Research and Practice [Software]. Bloomington/Albuquerque: Indiana University/SciTech Strategies. Available: https://sci2.cns.iu.edu

Jacomy, M., Venturini, T., Heymann, S. \& Bastian, M. (2014). ForceAtlas2, a continuous graph layout algorithm for handy network visualization designed for the Gephi software. PloS One, 9(6), 1-12. https://doi.org/10.1371/journal.pone.0098679 Johanson, J. \& Vahlne, J.-E. (2015). The Uppsala Internationalization Process Model Revisited: From Liability of Foreignness to Liability of Outsidership. In: M. Forsgren, U. Holm \& J. Johanson, Knowledge, Networks and Power (pp. 153186). London: Palgrave Macmillan. https://doi.org/10.1057/9781137508829_7 
Johanson, J. \& Vahlne, J. (1990). The Mechanism of Internationalisation. International Marketing Review, 7(4), 11-24. https://doi.org/10.1108/02651339010137414 Johanson, J. \& Vahlne, J.-E. (1977). The Internationalization Process of the Firm-A Model of Knowledge Development and Increasing Foreign Market Commitments. Journal of International Business Studies, 8, 23-32. https://oi.org/10.1057/palgrave.jibs.8490676

Landínez, D. A., Robledo, S. \& Montoya, D. M. (2019). Executive function performance in patients with obesity: a systematic review. Psychologia; an International Journal of Psychology in the Orient, 13(2), 121-134. https://doi. org/10.21500/19002386.4230

Larios, G. J. (2018). Patterns in international ICT entrepreneurship: Mexico's case. Academia Revista Latinoamericana de Administración, 31(4), 633-650. https:// doi.org/10.1108/ARLA-12-2016-0327

Lin, Y. (2015). Firm heterogeneity and location choice of Chinese firms in Latin America and the Caribbean: Corporate ownership, strategic motives and host country institutions. China Economic Review, 34(C), 274-292. https://doi.org/10.1016/j. chieco.2015.03.001

López, L. E., Kundu, S. K. \& Ciravegna, L. (2009). Born global or born regional? Evidence from an exploratory study in the Costa Rican software industry. Journal of International Business Studies, 40(7), 1228-1238. https://doi.org/10.1057/ jibs.2008.69

Luo, Y. \& Tung, R. L. (2007). International expansion of emerging market enterprises: A springboard perspective. Journal of International Business Studies, 38(4), 481-498. https://doi.org/10.1057/palgrave.jibs.8400275

Malhotra, S., Lin, X. \& Farrell, C. (2016). Cross-national uncertainty and level of control in cross-border acquisitions: A comparison of Latin American and U.S. multinationals. Journal of Business Research, 69(6), 1993-2004. https://doi. org/10.1016/j.jbusres.2015.10.145

Marín, J. C., Robledo, S. \& Duque-Méndez, N. D. (2017). Marketing Emprendedor: Una perspectiva cronológica utilizando Tree of Science. Revista Civilizar de Empresa y Economía, 7(13), 113-123. Available: https://revistas.usergioarboleda. edu.co/index.php/ceye/article/view/923

Martin, S. L. \& Javalgi, R. (2016). Entrepreneurial orientation, marketing capabilities and performance: The Moderating role of Competitive Intensity on Latin American International New Ventures. Journal of Business Research, 69(6), 2040-2051. https://doi.org/10.1016/j.jbusres.2015.10.149

Mathews, J. A. (2006). Dragon multinationals: New players in 21st century globalization. Asia Pacific Journal of Management, 23(1), 5-27. https://oi.org/10.1007/ s10490-006-6113-0

Mathieu, B., Sebastien, H. \& Mathieu, J. (2009). Gephi: an open source software for exploring and manipulating networks. [Software]. New York: International AAAI Conference on Weblogs and Social Media. Available: https://gephi.org/users/publications/ 
Moreira, M. Z. \& Ogasavara, M. H. (2018). Formal and informal institutions and the expatriation assignment: The case of Japanese subsidiaries in Latin America. Japan and the World Economy, 47, 18-26. https://doi.org/10.1016/j.japwor.2018.03.005

Newburry, W., Gardberg, N. A. \& Sánchez, J. I. (2014). Employer Attractiveness in Latin America: The Association Among Foreignness, Internationalization and Talent Recruitment. Journal of International Management, 20(3), 327-344. https://doi.org/10.1016/j.intman.2014.01.001

Ohri, A. (2012). $R$ for Business Analytics. Springer Science \& Business Media. New York: Springer-Verlag. https://doi.org/10.1007/978-1-4614-4343-8

Omokaro-Romanus, C., Anchor, J. R. \& Konara, P. (2019). The internationalization of Nigerian firms: Motivations and location patterns. Thunderbird International Business Review, 61(1), 75-88. https://doi.org/10.1002/tie.21962

Peng, M. W., Wang, D. Y. L. \& Jiang, Y. (2008). An institution-based view of international business strategy: a focus on emerging economies. Journal of International Business Studies, 39(5), 920-936. https://doi.org/10.1057/palgrave. jibs.8400377

Pourkhani, A., Abdipour, K., Baher, B. \& Moslehpour, M. (2019). The impact of social media in business growth and performance: A scientometrics analysis. International Journal of Data and Network Science, 3, 223-244. https://doi. org/10.5267/j.ijdns.2019.2.003

Puck, J. \& Filatotchev, I. (2018). Finance and the multinational company: Building bridges between finance and global strategy research. Global Strategy Journal, 10(4), 655-675. https://doi.org/10.1002/gsj.1330

Reuber, A. R., Dimitratos, P. \& Kuivalainen, O. (2017). Beyond categorization: New directions for theory development about entrepreneurial internationalization. Journal of International Business Studies, 48(4), 411-422. https://doi.org/10.1057/ s41267-017-0070-3

Robledo, S., Osorio, G. \& López, C. (2014). Networking en pequeña empresa: una revisión bibliográfica utilizando la teoria de grafos. Vínculos, 11(2), 6-16. Disponible en https://revistas.udistrital.edu.co/index.php/vinculos/article/view/9664

Rugman, A. M. \& Verbeke, A. (2004a). Towards a Theory of Regional Multinationals: A Transaction Cost Economics Approach. In: K. Macharzina (Ed.), The Limits to Globalization and the Regional Strategies of Multinational Enterprises (pp. 5-17). Gabler Verlag: Springer Fachmedien Wiesbaden. https://doi. org/10.1007/978-3-322-91005-9_1

Rugman, A. M. \& Verbeke, A. (2004b). A perspective on regional and global strategies of multinational enterprises. Journal of International Business Studies, 35(1), 3-18. https://doi.org/10.1057/palgrave.jibs.8400073

Taiebi Javid, E., Nazari, M. \& Ghaeli, M. R. (2019). Social media and e-commerce: A scientometrics analysis. International Journal of Data and Network Science, (3), 269-290. https://doi.org/10.5267/j.ijdns.2019.2.001

Tani, M., Papaluca, O. \& Sasso, P. (2018). The System Thinking Perspective in the Open-Innovation Research: A Systematic Review. Journal of Open Innovation: 
Technology, Market, and Complexity, 4(3), 1-28. https://doi.org/10.3390/joitmc4030038

Varma, S., Bhasin, N. \& Nayyar, R. (2015). Motives as Locational Determinants: A Study of FDI between India and LAC. Transnational Corporations Review, 7(3), 335-352. https://doi.org/10.5148/tncr.2015.7306

Vélez-Calle, A., Sánchez-Henríquez, F. \& Contractor, F. (2018). Internationalization and performance: the role of depth and breadth. Academia Revista Latinoamericana de Administración, 31(1), 91-104. https://doi.org/10.1108/ARLA-04-20170125

Vendrell-Herrero, F., Gomes, E., Mellahi, K. \& Child, J. (2017). Building international business bridges in geographically isolated areas: The role of foreign market focus and outward looking competences in Latin American SMEs. Journal of World Business, 52(4), 489-502. https://doi.org/10.1016/j.jwb.2016.08.007

Wallis, W. D. (2007). A Beginner's Guide to Graph Theory. Boston: Birkhäuser Basel. https://doi.org/10.1007/978-0-8176-4580-9

Wasserman, S. \& Faust, K. (2012). Social Network Analysis: Methods and Applications. Cambridge: Cambridge University Press. https://oi.org/10.1017/ CBO9780511815478

Wiersema, M. F. \& Bowen, H. P. (2011). The relationship between international diversification and firm performance: Why it remains a puzzle. Global Strategy Journal, 1(1-2), 152-170. https://doi.org/10.1002/gsj.5

Wright, M., Filatotchev, I., Hoskisson, R. E. \& Peng, M. W. (2005). Strategy Research in Emerging Economies: Challenging the Conventional Wisdom*. Journal of Management Studies, 42(1), 1-33. https://doi.org/10.1111/j.1467-6486.2005.00487.x

Wu, J., Pangarkar, N. \& Wu, Z. (2016). The moderating effect of technology and marketing know-how in the regional-global diversification link: Evidence from emerging market multinationals. International Business Review, 25(6), 12731284. https://doi.org/10.1016/j.ibusrev.2016.03.012

Yang, S., Keller, F. B. \& Zheng, L. (2016). Social Network Analysis: Methods and Examples. New York: SAGE Publications. https://dx.doi.org/10.4135/9781071802847

Yaprak, A. \& Karademir, B. (2010). The internationalization of emerging market business groups: an integrated literature review. International Marketing Review, 27(2), 245-262. https://doi.org/10.1108/02651331011037548

Zaheer, S. (1995). Overcoming the liability of foreignness. Academy of Management Journal. Academy of Management, 38(2), 341-363. Available: https://www.jstor. org/stable/256683

Zhang, J. \& Luo, Y. (Mar. 2017). Degree Centrality, Betweenness Centrality, and Closeness Centrality in Social Network. Proceedings of the 2017 2nd International Conference on Modelling, Simulation and Applied Mathematics (MSAM2017). Bangkok, Thailand. https://doi.org/10.2991/msam-17.2017.68

Zuluaga, M., Robledo, S., Osorio, G. A., Yathe, L., González, D. \& Taborda, G. (2016). Metabolómica y Pesticidas: Revisión sistemática de literatura usando teoría de grafos para el análisis de referencias. Nova, 14(25), 121. https://doi. org/10.22490/24629448.1735 
Zupic, I. \& Čater, T. (2015). Bibliometric Methods in Management and Organization. Organizational Research Methods, 18(3), 429-472. https://doi. org/10.1177/1094428114562629

\section{BiodATA}

Pedro Luis Duque Hurtado es estudiante del doctorado en Administración en la Universidad Nacional de Colombia (Sede Manizales). Magister en Administración de la Universidad Nacional de Colombia (Sede Manizales). Administrador de empresas de la Universidad Nacional de Colombia (Sede Manizales). Docente de la facultad de Ciencias Administrativas, Económicas y Contables de la Universidad Católica Luis Amigo (Colombia). https://orcid.org/0000-0003-4950-8262

Oscar Eduardo Meza Aguirre es Economista de la Universidad de Manizales (Colombia). Magister en Administración de la Universidad Nacional de Colombia, y candidato a doctor en sostenibilidad y desarrollo de la Universidad de Manizales. Sus intereses incluyen la investigación en negocios e internacionales, comercio, internacionalización de organizaciones, estrategia y desarrollo sostenible. Es investigador de la Universidad Nacional de Colombia (Sede Manizales) y en la Universidad Católica Luis Amigó (Manizales, Colombia). https://orcid.org/0000-0002-1198-5955

Gastón Adolfo Zapata Lesmes es Doctor en Educación por la Universidad Central de Nicaragua. Master en Master in English Didactic por la Universidad de Caldas (Colombia), Master in Education and Human Development por la Universidad de Manizales (Colombia). Más de 24 años de experiencia en educación, enseñanza del inglés como lengua extranjera en los niveles de educación media, pregrado y posgrado. Evaluador externo de proyectos de investigación. Docente cursos de investigación. Asesor de tesis de Pregrado. Docente Investigador en estrategias del aprendizaje del inglés desde hispanohablantes. Investigador en desarrollo de competencias lectoras básicas en inglés en cursos de ESP. Director Académico y Administrativo Instituto Meyer de Colombia. Director del departamento de Idiomas de la Universidad de Manizales. https://orcid.org/0000-0002-8869-7644

José David Giraldo Castellanos es Administrador de Empresas y Magíster en Administración de la Universidad Nacional de Colombia. Los campos interés académico y profesional, se orientan a investigaciones sobre temáticas de comercio exterior, negocios internacionales, macroeconomía y estrategias de negocio. https://orcid. org/0000-0002-1278-8697 